\title{
Influence of Tempered Microstructures on the Transformation Behaviour of Cold Deformed and Intercritically Annealed Medium Carbon Low Alloy Steel
}

\author{
Kenneth Kanayo Alaneme* \\ Department of Materials Engineering, Indian Institute of Science, Bangalore, India
}

Received: November 19, 2009; Revised: March 4, 2010

\begin{abstract}
This research is focused on understanding the role of microstructural variables and processing parameters in obtaining optimised dual phase structures in medium carbon low alloy steels. Tempered Martensite structures produced at 300,500 , and $650{ }^{\circ} \mathrm{C}$, were cold rolled to varied degrees ranging from 20 to $80 \%$ deformation. Intercritical annealing was then performed at 740,760 , and $780{ }^{\circ} \mathrm{C}$ for various time duration ranging from 60 seconds to 60 minutes before quenching in water. The transformation behaviour was studied with the aid of optical microscopy and hardness curves. From the results, it is observed that microstructural condition, deformation, and intercritical temperatures influenced the chronological order of the competing stress relaxation and decomposition phase reactions which interfered with the rate of the expected $\alpha \rightarrow \gamma$ transformation. The three unique transformation trends observed are systematically analyzed. It was also observed that the 300 and $500{ }^{\circ} \mathrm{C}$ tempered initial microstructures were unsuitable for the production of dual structures with optimized strength characteristics.
\end{abstract}

Keywords: dual phase structure, stress relaxation, intercritical annealing, tempered martensite

\section{Introduction}

This work is a contribution to existing knowledge on the understanding of the transformation mechanisms associated with the formation of viable dual phase microstructures in medium carbon low alloy steels. Dual phase steels have found continued relevance in the automobile industries because of its unique high strength to weight ratio, formability, energy savings and crash energy management ${ }^{1-10}$. This has made them suitable for the production of car body parts, wheel rims, and a good number of structural parts. The dual phase steels consist of a ferrite (soft phase) and martensite (hard phase) composite structure, which is responsible for its unique mechanical properties. Initially, dual phase steels were technically defined as steels with microstructure consisting of $30 \%$ martensite with the balance ferrite; but as a result of its amazing combination of mechanical properties, there has also been interest in the development of dual phase steels with between $50-60 \%$ martensite derived by the utilization of steels of medium carbon composition. Dual phase steels with $50-60 \%$ martensite have been touted to have the potentials for good combination of high strength, toughness, ductility and fatigue strength - extending the use of dual phase steels for more structural applications $\mathrm{s}^{6,11-12}$. The steel chemistry and processing parameters such as intercritical temperature, soaking time, cooling rate, and deformation processes; have been observed to influence the proportion, dispersion and morphology of the dual phase microstructures ${ }^{2,13-17}$. The effects of these parameters are better understood and well documented for dual phase steels with carbon contents less than 0.2 weight $\%{ }^{15-18}$. However for steels with carbon contents in the near medium carbon classification range $-0.2 \% \mathrm{C}-0.3 \% \mathrm{C}$, where the effect of carbon during transformation starts becoming more pronounced, it has been a little difficult controlling and comprehending the transformation processes occurring during the expected $\alpha \rightarrow \gamma$ transformation in the intercritical phase domain. This has made it difficult to control effectively the grain sizes, phase distribution and morphology which are crucial for obtaining optimized properties ${ }^{19-22}$. This is due to other competing metallurgical phase reactions like solid solution decomposition, precipitation, recrystallization and coalesces of phases; which like crystallographic phase changes, require defect structures (potential nucleation sites) to occur. Thus the phase reactions often occur concurrently or mutually retard each other with the seeming confusing order of the transformations, making the overall mechanism difficult to analyze and control ${ }^{23-28}$. In the present work, the role of the starting point microstructures - in this case tempered martensite microstructures, plastic deformation, intercritical temperatures and soaking time on the transformation behaviour of medium carbon low alloy steels is investigated. The steel grades have recently been considered for the fabrication of improvised engine seat protectors and bumpers by indigenous automobile technicians and artisans. The conventionally processed grades were found to have adequate tensile strength, toughness, and fatigue strength but limited formability which informed the choice of dual phase processing.

\section{Materials and Method}

The chemical composition of the steel employed for this research was Fe $0.3 \mathrm{C}-0.26 \mathrm{Si}-1.19 \mathrm{Mn}-0.0419 \mathrm{P}-0.0024 \mathrm{~S}-0.13 \mathrm{Cr}$ $-0.035 \mathrm{Ni}-0.0017 \mathrm{~V}$. It was supplied in the form of hot rolled plates $14 \mathrm{~mm}$ thick. The plates were cut and machined to flat strips of thickness ranging from 2.5 to $10 \mathrm{~mm}$. Three tempered martensitic structures were produced on different sets of strips for use as starting point microstructures. The essence was to achieve carbide precipitates of different sizes and dispersion. The tempering treatments were performed by austenitizing the strips at $860^{\circ} \mathrm{C}$ for one hour and then quenching in water. Then tempering was performed at three different sub-critical temperatures of 300,500 , and $650{ }^{\circ} \mathrm{C}$; soaking for one hour and then cooled in air. The ensuing tempered microstructures were designated M300, M500, and M650 to indicate that tempering 
was performed at temperatures of 300,500 , and $650{ }^{\circ} \mathrm{C}$ respectively. Cold rolling of the different starting point microstructures was performed using a laboratory cold rolling machine. The strips were deformed to various degrees of $20,50,70$ and $80 \%$ with a final uniform thickness of $2 \mathrm{~mm}$ for each strip. The strips were cut to sizes $10 \times 10 \times 2 \mathrm{~mm}$ for inter critical annealing. The intercritical annealing treatment was performed at temperatures of 740, 760, and $780^{\circ} \mathrm{C}$; and the samples were held at each temperature for different time durations ranging from 30 seconds to 60 minutes before water quenching. The hardness of each intercritical annealed sample was evaluated using a Rockwell hardness Tester using a ' $\mathrm{C}$ ' scale. Three to four repeat tests were performed on each sample and the average taken as a measure of the hardness of the representative structure (specimen). The hardness values were plotted against time values to aid analysis of the transformation behaviour of the test specimens with respect to the processing conditions. Microstructural characterization of the test specimens was performed using an optical microscope with accessories for image analyses. SigmaScanpro software for image analysis was utilized for estimation of volume fraction of phases, and average sizes of the cementite precipitates observed. The specimen preparation and etching was performed following standard procedures in accordance with ${ }^{29}$.

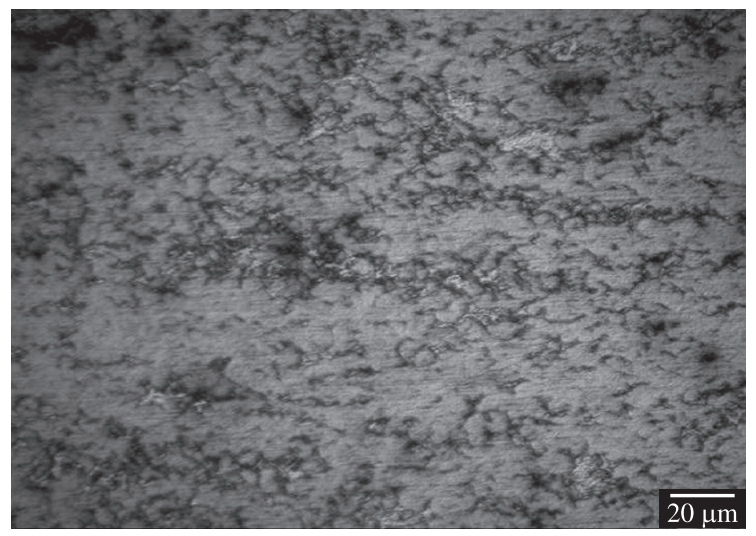

(a)

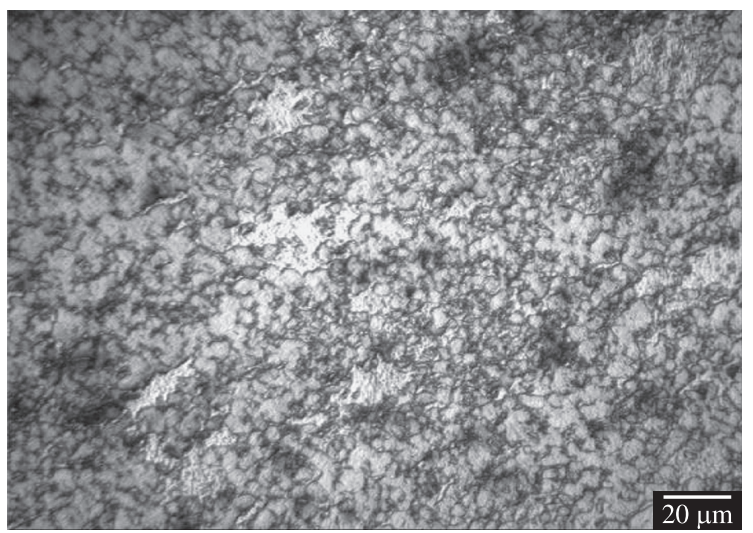

(c)

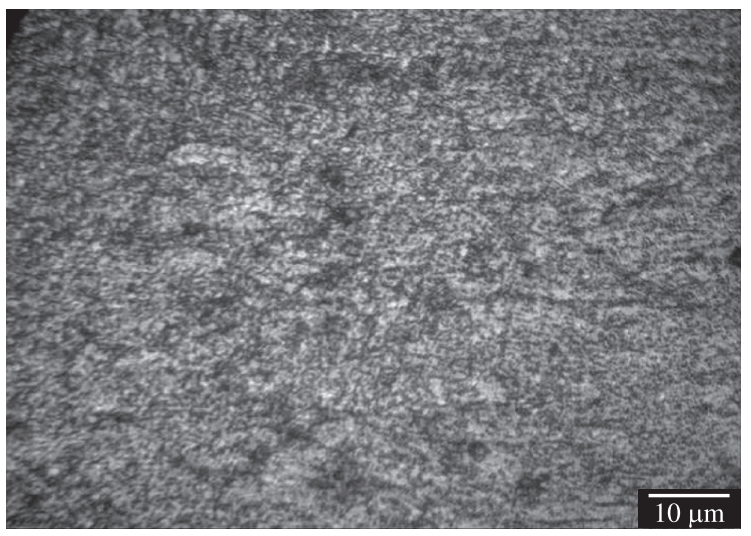

(b)

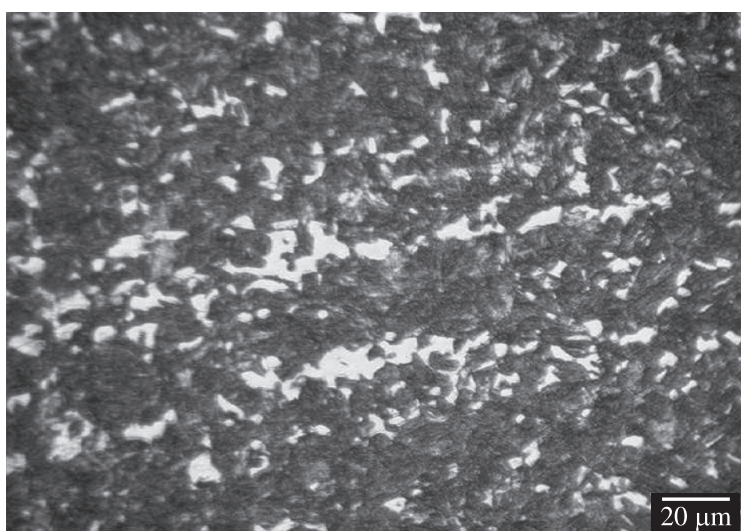

(d)

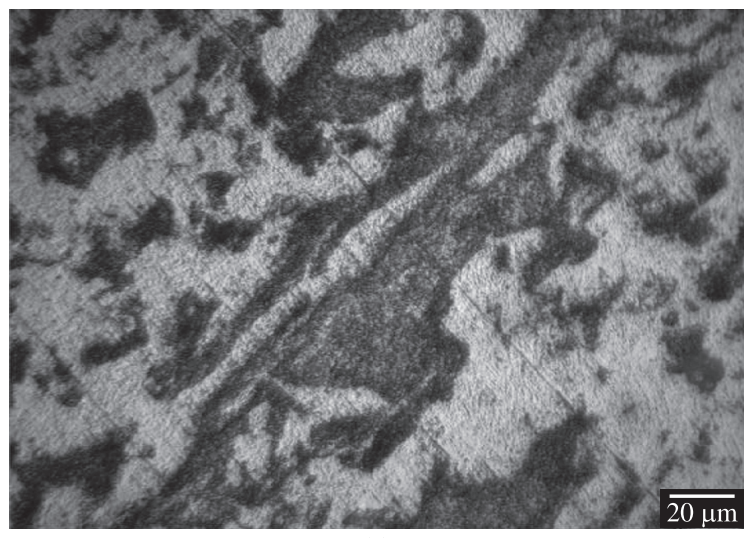

(e)

Figure 1. a) As-deformed structure of M500 showing conspicuous deformation bands revealing the direction of cold rolling; b) M500 structure after 1 minute of treatment at $740{ }^{\circ} \mathrm{C}$. The structure reveals fine precipitation of cementite; c): M500 structure after 15 minutes of treatment at $740{ }^{\circ} \mathrm{C}$. The structure reveals coarse cementite particles and some recrystallization nuclei; d) M500 structure after 30minutes of treatment at $740{ }^{\circ} \mathrm{C}$. The structure consists of ferrite (white phase) and martensite (dark phase); and e) M500 structure after 1hour of treatment at $740{ }^{\circ} \mathrm{C}$. The structure consists of large dispersed phase of martensite (dark phase) in continuous phase of ferrite (gray phase). 


\section{Results and Discussion}

\subsection{Transformation mechanisms}

Figure 1 show the microstructures representative of the transformation stages for all tempered structures intercritically annealed at $740{ }^{\circ} \mathrm{C}$. Alaneme and $\mathrm{Kamma}^{30}$ had earlier idealized the transformation sequence which was for most experimental observation for the 740 and $760{ }^{\circ} \mathrm{C}$ intercritical annealing treatment, observed to progress in 4 transformation stages. The four stages are namely - recovery processes resulting in a slight drop in hardness (stage 1); primary recrystallization of ferrite, intense spheroidization and decomposition of cementite resulting in a sharp drop in hardness (stage 2), $\alpha \rightarrow \gamma$ transformation resulting in martensite formation on quenching leading to sharp rise in hardness (stage 3), and grain growth of the recrystallized ferrite and transformed austenite grains leading to reduced hardness on quenching (stage 4). This idealized transformation sequence is consistent with the microstructural observations shown in Figure 1. Figure 1a shows the as-deformed structure with conspicuous deformation bands depicting the direction of cold rolling. Figure $1 \mathrm{~b}$ shows the deformed structure after 1 minute of soaking (of stage 1), in which it is observed that there is fine precipitation of cementite with grain size averagely $800 \mathrm{~nm}$ (recovery). The structure after soaking for 15 minutes is shown in Figure 1c - a much coarser cementite structure having average grain size of $4 \mu \mathrm{m}$ is observed which resulted in a drastic reduction in hardness, marking stage 2 . Figure $1 \mathrm{~d}$ shows the structure after soaking for 30 minutes - the structure consists of about 42 volume percent ferrite (white phase) and 58 volume percent martensite (dark phase) indicating stage 3 transformation; while Figure 1e shows the structure obtained after soaking for 60 minutes - the structure has a much larger grain size of ferrite (white phase) and martensite (dark phase) indicating stage 4 transformation.

\subsection{Influence of percent deformation}

The hardness variations for the tempered specimens intercritically annealed at $740{ }^{\circ} \mathrm{C}$ are shown in Figure 2. From Figure 2a it is observed that regardless of the degree of deformation, the M300 specimens deviated from the idealized transformation sequence as there was prolonged stage 1 (recovery) characterized by a slight drop in hardness and absence of stage 2 transformation in comparison with the M500 (Figure 2b) and the M650 (Figure 2c) specimens. It is also observed that peak hardness was not attained after 60 minutes soaking time prior to quenching in water for all specimens. The observed trend is indicative of the fact that the partial austenitization process needed for the formation of dual phase structures is sluggish at $740{ }^{\circ} \mathrm{C}$ when the M300 specimens are utilized. The trend is attributed to the higher meta-stable structure produced at $300{ }^{\circ} \mathrm{C}$ which undergoes further precipitation and growth of cementite which is thermally favoured ahead of the expected decomposition of cementite and some ferrite for austenite formation - thus the observed delay in the $\alpha \rightarrow \gamma$ transformation (stage 3). Kamma and Horbogen ${ }^{23}$ have reported a similar behaviour in subcritically processed steel in which it was stated that the M300 structure undergoes further precipitation of cementite which was incomplete in the course of preliminary tempering heat-treatment and thus continuous reaction (subgrain growth) was the recrystallization mechanism predominant. The M500 on the other hand (Figure 2b) is observed to follow the idealized transformation sequence, especially for the heavily prior deformed specimens (70 and $80 \%$ deformed specimens). It is observed that the lightly prior deformed specimens (20 and $50 \%$ prior deformation) had a slightly slower transformation rate as stage 4 is not attained for both degrees of deformation. It is also observed that the intensity
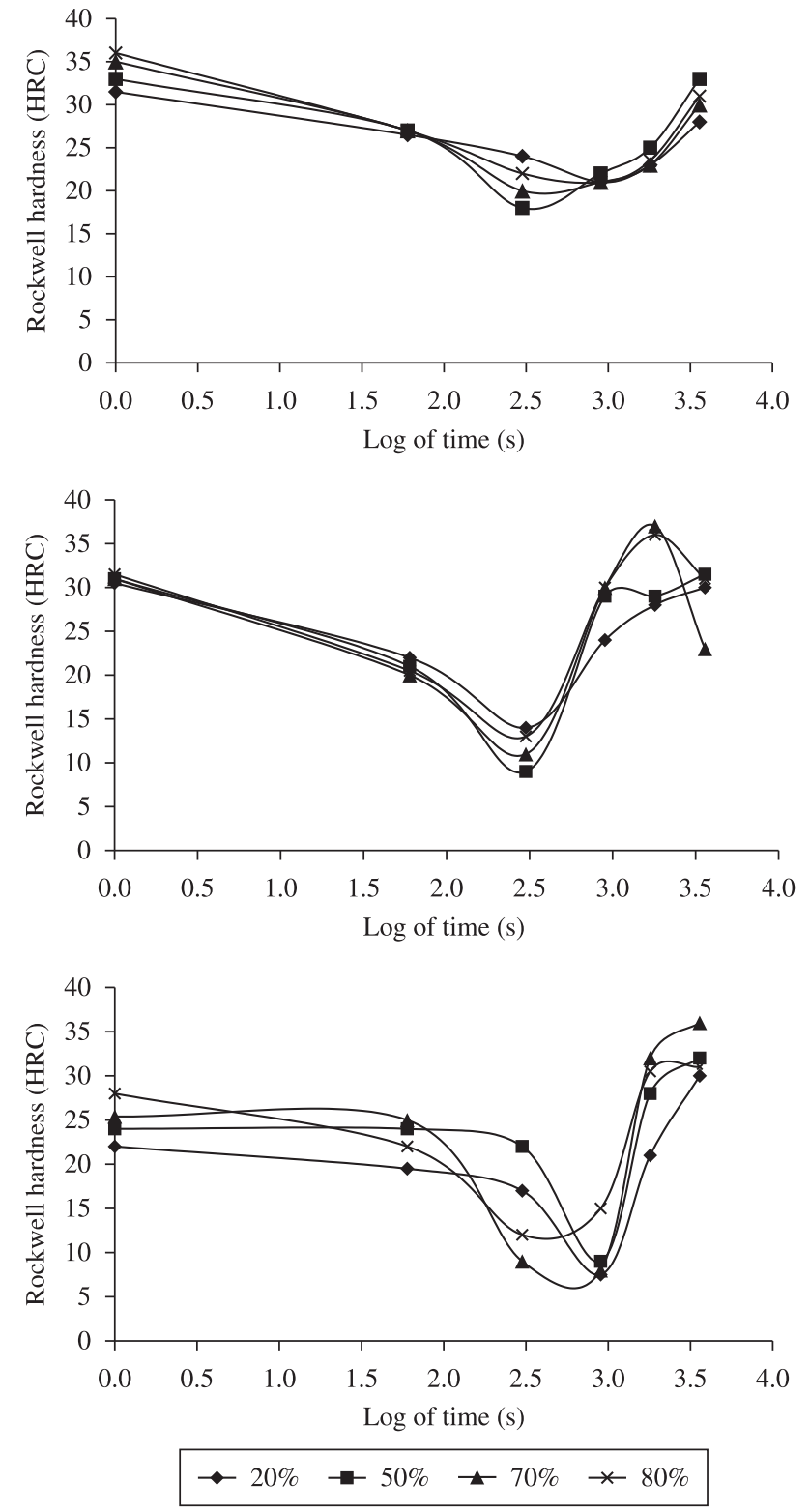

Figure 2. Rockwell hardeness vs. Annealing time plot for: a) $300{ }^{\circ} \mathrm{C}$ tempered martensite specimens treated at $740{ }^{\circ} \mathrm{C}$; b) $500{ }^{\circ} \mathrm{C}$ tempered martensite specimens treated at $740{ }^{\circ} \mathrm{C}$ and c) $650{ }^{\circ} \mathrm{C}$ tempered martensite specimens treated at $740{ }^{\circ} \mathrm{C}$.

of stage 2 (spheroidization and decomposition of cementite and recrystallization of ferrite) is less for the $20 \%$ deformed specimen. This can be attributed to a lesser dislocation density, defect structures and stored energy needed to facilitate the transformation process ${ }^{24}$. The M650 also follows the idealized transformation sequence (Figure 2c) but it is observed that for the lightly deformed samples, there is a prolonged stage 1 in comparison with the 70 and $80 \%$ deformed specimens. Also in comparison to the M500 (Figure 2b), it is observed that the onset of stage 2 for the M650 specimens is longer. The delay in the commencement of stage 2 can be attributed to the fact that the M650 structure is more stable; and hence the driving force for decomposition of the phases is low. Thus at $740{ }^{\circ} \mathrm{C}$ the overall effect of the degree of deformation is significant especially for the M500 and M650 specimens. 

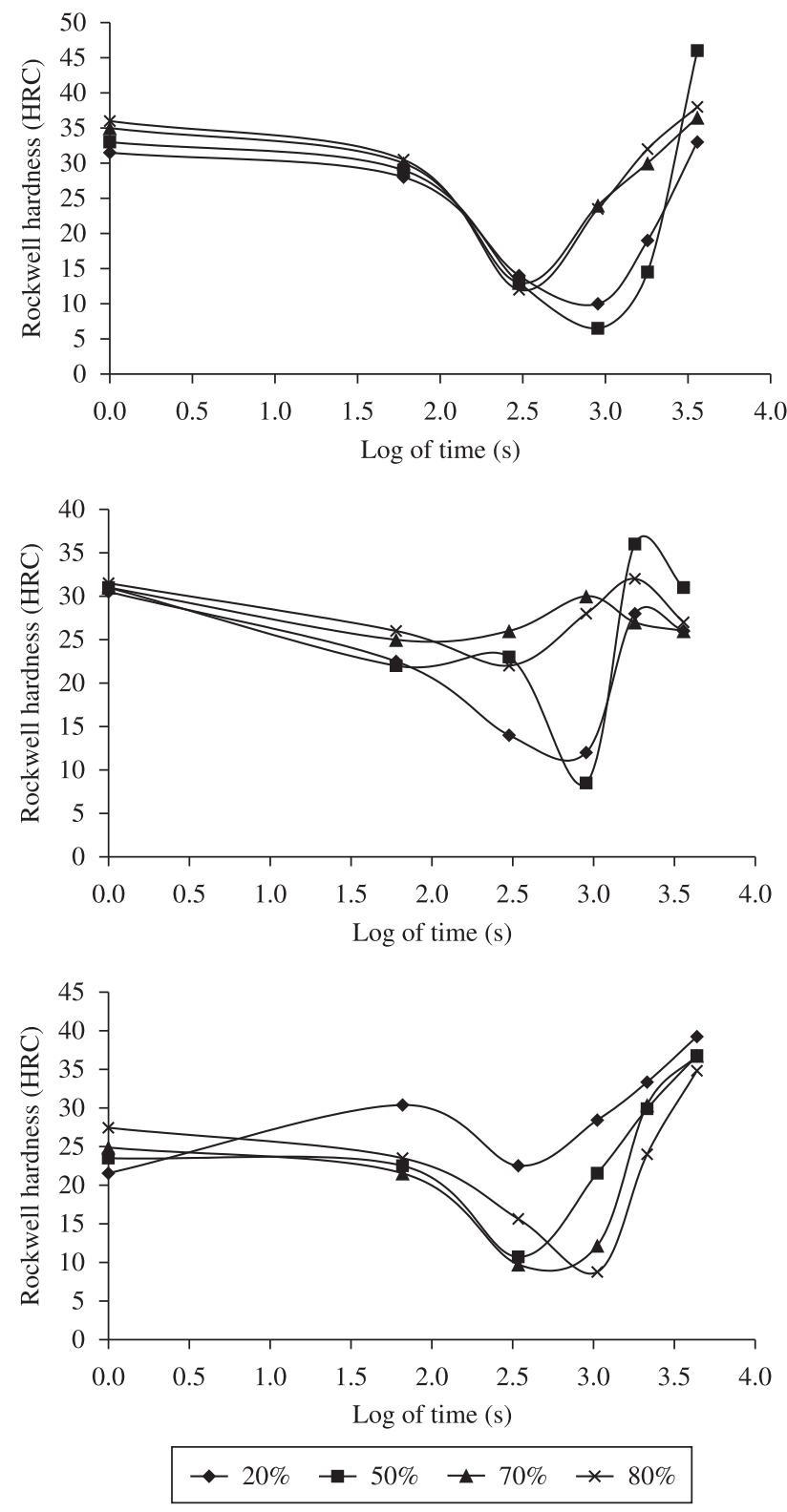

Figure 3. Rockwell hardeness vs. Annealing time plot for: a) $300^{\circ} \mathrm{C}$ tempered martensite specimens treated at $760{ }^{\circ} \mathrm{C}$; b) $500{ }^{\circ} \mathrm{C}$ tempered martensite specimens treated at $760{ }^{\circ} \mathrm{C}$ and c) $6500^{\circ} \mathrm{C}$ tempered martensite specimens treated at $760{ }^{\circ} \mathrm{C}$.

A trend consistent with the observations for intercritical annealing at $740{ }^{\circ} \mathrm{C}$ is also observed for the tempered specimens treated at $760{ }^{\circ} \mathrm{C}$, as shown in Figure 3. The M300 specimens in this case (Figure 3a) conform more to the idealized transformation sequence as there is a more defined stage 2 in comparison to treatment at $740{ }^{\circ} \mathrm{C}$ Figure 2(a), though the stage 4 transformation is not attained - indicating a sluggish transformation similar to that observed for intercritical annealing at $740{ }^{\circ} \mathrm{C}$. Also, it is observed that the onset of stage 3 is faster for the heavily prior deformed samples (70 and $80 \%$ ) than the 20 and $50 \%$ deformed samples. Again peak hardness is not attained indicating that the transformation is generally slow. For the M500 specimens (Figure 3b), there are marked deviation from the idealized transformation sequence for the heavily prior deformed samples - that is the 70 and $80 \%$ deformation in comparison to the
20 and $50 \%$ deformed samples. This can be attributed to the very fast $\alpha \rightarrow \gamma$ transformation which apparently occurs concurrently with the recrystallization and decomposition process making the decomposition of the old phase (stage 2) less apparent in comparison with the 20 and $50 \%$ deformed specimens. It is also observed that peak hardness is attained for all prior deformed specimens. For the M650 samples, it is observed that the transformation rate is slower in comparison to the M500 as peak hardness is not attained for all degrees of prior deformation.

The transformation sequence at $780^{\circ} \mathrm{C}$ for the tempered structures exhibits the five stage and three stage transformation model as reported by Alaneme and $\mathrm{Kamma}^{30}$ for normalized structures. The general feature of the two transformation models is the absence of stage 2 (primary recrystallization of ferrite and intense spheroidization of cementite) of the four stage transformation sequence for intercritical annealing at 740 and $760{ }^{\circ} \mathrm{C}$, which resulted in a sharp reduction in hardness. The higher intercritical temperature of $780{ }^{\circ} \mathrm{C}$ leads to the availability of adequate energy needed to overcome the activation energy barriers of the competing transformation processes. Hence, the decomposition of cementite precipitates and the $\alpha \rightarrow \gamma$ transformation occurs concurrently. Thus the softening created by the decomposition of the cementite precipitates is counteracted by the simultaneous production of the hard martensitic structure which is developed on quenching the specimen in water. Similar transformation behaviour has been observed in a couple of two phase metallic systems ${ }^{20,27,31}$.

In the case of the M300 (Figure 4a), there is no deducible relationship between the degree of deformation and the consequent transformation sequence. It is observed that the 50 and $70 \%$ deformed specimens followed the five stage transformation sequence (Stage I - stress relief (recovery), characterised by a slight drop in hardness; Stage II - recrystallization of both $\alpha$ and $\gamma$, characterised by a sharp rise in hardness; Stage III - grain growth of recrystallized $\alpha$ grains which is competing with further $\gamma$ nucleation; causing arrest in increase in hardness; Stage IV - further $\gamma$ nucleation and homogenization causing further increase in hardness due to more martensite formation; and Stage $\mathrm{V}$ - grain growth of $\alpha$ and $\gamma$ grains resulting in loss of hardness). The 20 and $80 \%$ deformed samples followed a three stage transformation sequence (Stage I - stress relief, characterised by a slight drop in hardness; Stage II recrystallization of both $\alpha$ and $\gamma$ accompanied with homogenization of $\gamma$, causing increased hardenability and more martensite formation, and characterised by increase in hardness and Stage III - grain growth of $\alpha$ and $\gamma$ characterised by drop in hardness). In the case of M500 (Figure 4b) it can still not be clearly ascertained if the degree of prior deformation influences the particular transformation sequence - of significance is the fact that higher hardness levels are obtained from the low prior deformed samples (20 and 50\% deformed) in comparison to the heavily deformed (70 and $80 \%$ deformation). For the M650, the three transformation models are observed - the 50 and $70 \%$ prior deformed samples followed the four stage transformation sequence discussed earlier for samples treated at 740 and $760{ }^{\circ} \mathrm{C}$; while the 20 and $80 \%$ deformed samples followed respectively the five stage and three stage model. Again the role of prior deformation is not easy to link to the observed multiple transformation models.

\subsection{Influence of temperature}

The influence of temperature on the transformation behaviour of the tempered specimens is shown in Figure 5. From Figure 5a, it is observed that the transformation kinetics is faster at $780{ }^{\circ} \mathrm{C}$ in comparison to 740 and $760{ }^{\circ} \mathrm{C}$. Also, it is observed that for annealing at $780{ }^{\circ} \mathrm{C}$ resulted in concurrent decomposition of cementite and $\alpha \rightarrow \gamma$ transformation which is why there is no sharp drop in hardness as compared to the 740 and $760{ }^{\circ} \mathrm{C}$ transformation sequence. For 

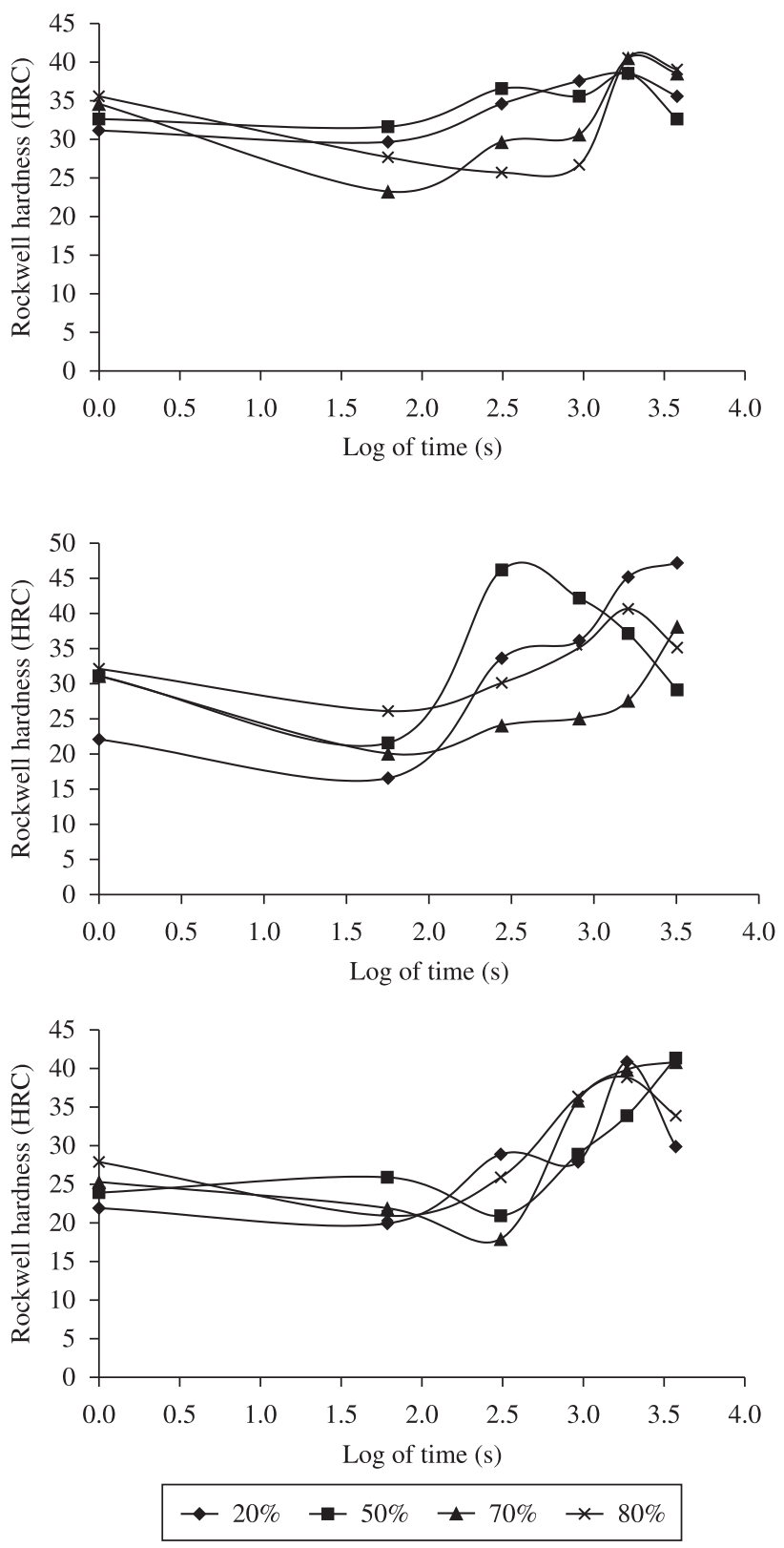

Figure 4. Rockwell hardeness vs. Annealing time plot for: a) $300^{\circ} \mathrm{C}$ tempered martensite specimens treated at $780{ }^{\circ} \mathrm{C}$; b) $500{ }^{\circ} \mathrm{C}$ tempered martensite specimens treated at $780{ }^{\circ} \mathrm{C}$ and c) $650{ }^{\circ} \mathrm{C}$ tempered martensite specimens treated at $780{ }^{\circ} \mathrm{C}$.

the M500 specimens, it is observed that the transformation rate is faster at $780{ }^{\circ} \mathrm{C}$ in comparison to 740 and $760{ }^{\circ} \mathrm{C}$. Also for the M650 specimens, it is observed that the transformation rate and transformation sequence is different for the $780{ }^{\circ} \mathrm{C}$ specimens in comparison to 740 and $760{ }^{\circ} \mathrm{C}$.

\subsection{Duplex microstructures}

Figure 6 shows representative microstructures produced revealing the influence of the initial microstructures on the formation of well defined duplex microstructures. From the micrographs, it is observed that the M300 and M500 structures yielded duplex structures with considerable amounts of secondary recrystallized grains in comparison with the M650 specimens. This is attributed to the
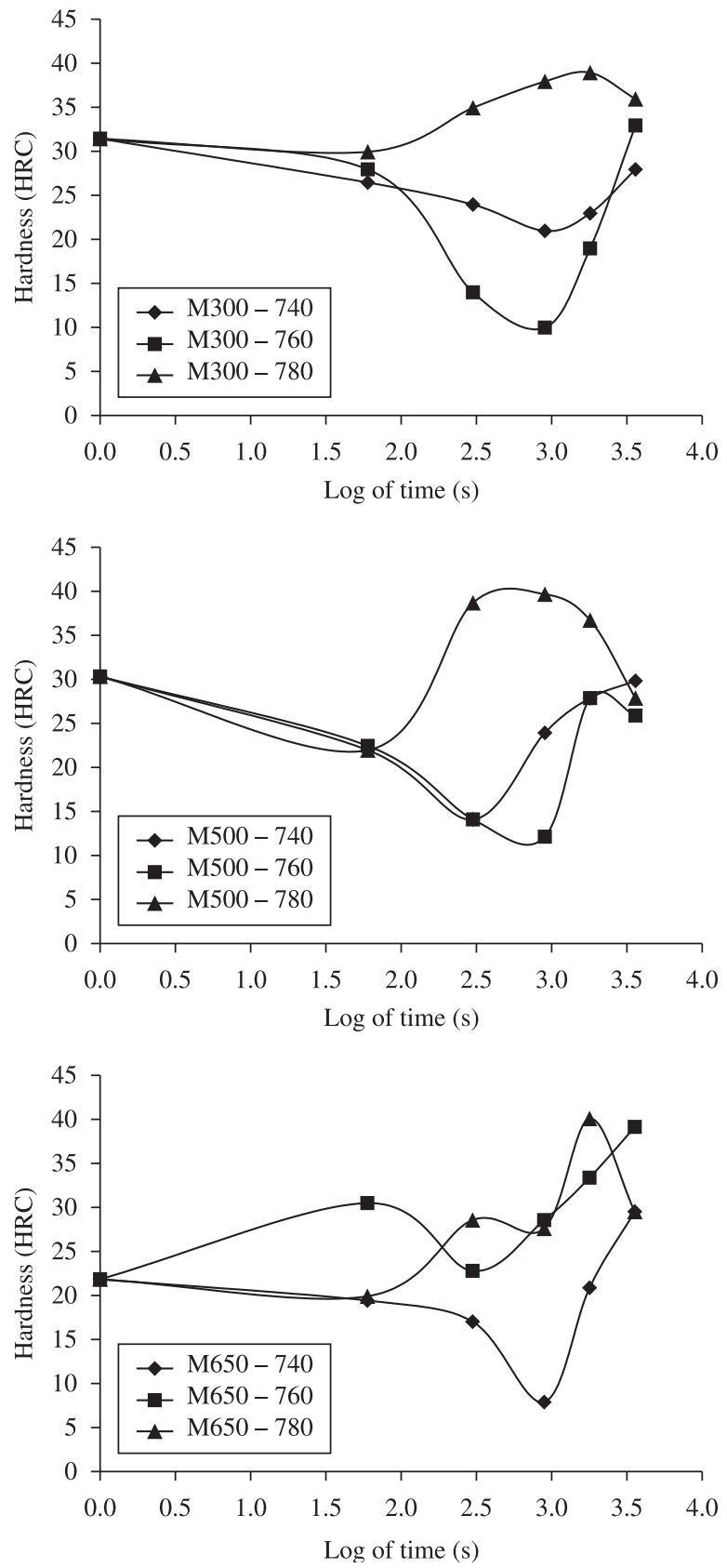

Figure 5. Variation of hardness with intercritical temperature for: a) M300 specimens, b) M500 specimens and c) M650 specimens.

competing precipitation and $\alpha \rightarrow \gamma$ transformation which retards the growth of primary recrystallized nuclei in regions in which cementite precipitation is still intense, as the precipitates pin and immobilize the recrystallization fronts ${ }^{24}$. Gorelik ${ }^{31}$ reported that portions in a microstructure with less intense precipitation favour the rapid growth of the primary recrystallized nuclei leading to grain inequigranularity. Thus a homogenized structure of ferrite and Martensite can only be achieved with much longer soaking time which will remove the inequigranularity but will invariably lead to reduced strength and strain hardening potentials because of the relative larger grain sizes in comparison to the M650 specimens. The relatively homogeneous duplex structures observed for the M650 specimens is due to the greater microstructural stability achieved by tempering at $650{ }^{\circ} \mathrm{C}$, 


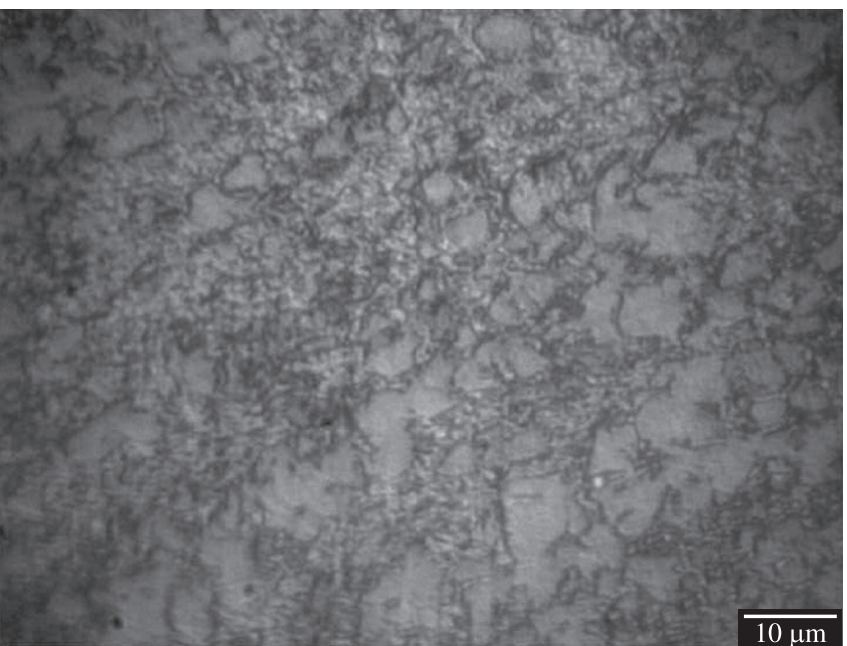

(a)

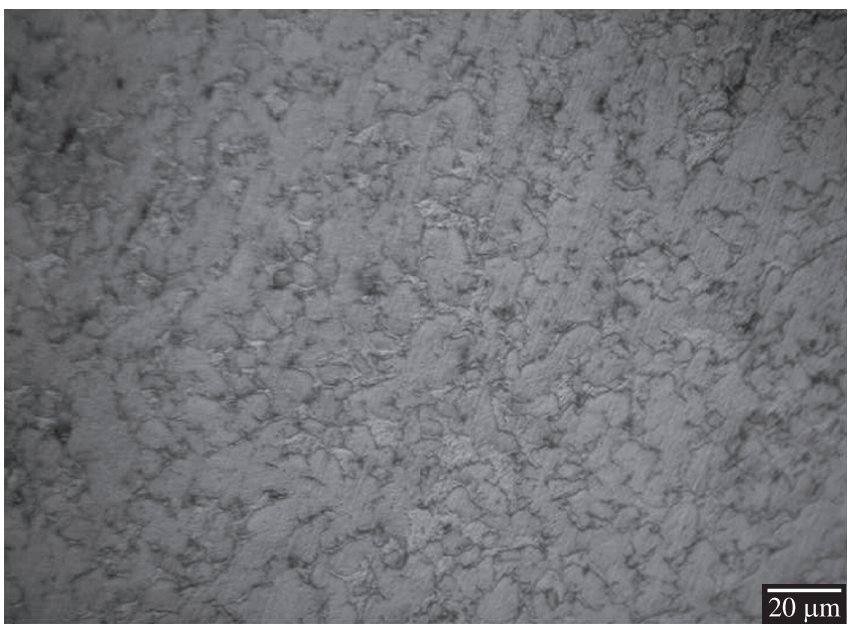

(b)

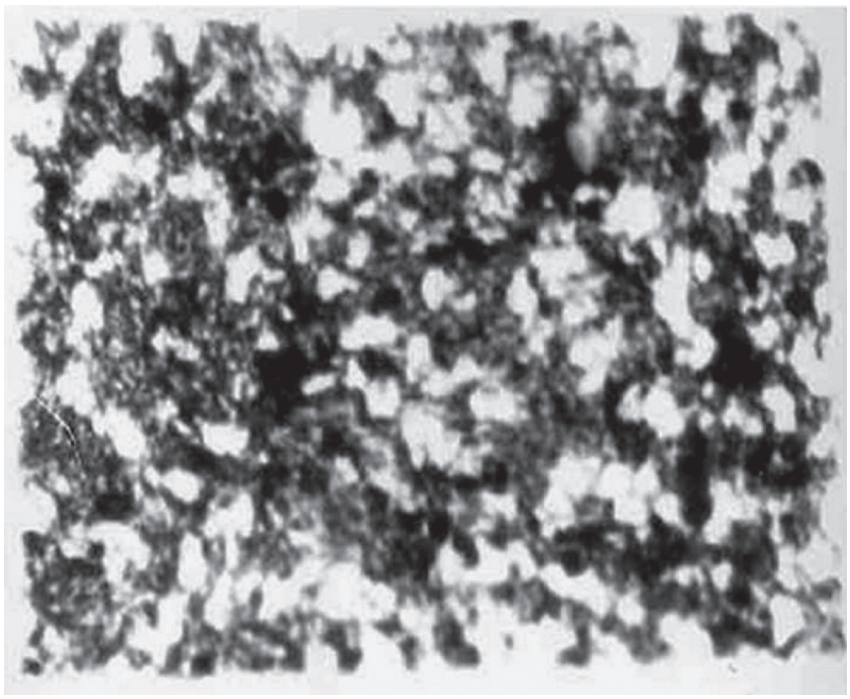

(c)

Figure 6. a) The microstructure of $\mathrm{M} 300 / 70 \% / 760{ }^{\circ} \mathrm{C}, 30 \mathrm{~min} / \mathrm{H}_{2} \mathrm{O}$. The structure reveals secondary recrystallized grains of ferrite with fine cementite particles; $b$ ) The microstructure of $\mathrm{M} 500 / 70 \% / 760{ }^{\circ} \mathrm{C}, 30 \mathrm{~min} / \mathrm{H}_{2} \mathrm{O}$. The structure reveals large dispersion of secondary recrystallized grains of ferrite; and c) The microstructure of $\mathrm{M} 650 / 70 \% / 760{ }^{\circ} \mathrm{C}, 30 \mathrm{~min} / \mathrm{H}_{2} \mathrm{O}$. The structure reveals well defined duplex microstructure of ferrite (white phase) and martensite (dark phase). as there is less precipitation from the structure during the of the intercritical annealing treatment ${ }^{14,23}$. This same phenomenon was observed for normalized structures which yielded very well defined equi-axed duplex structures with optimized strength and plasticity characteristics, which was attributed to the stable nature of the normalized structures ${ }^{12,14,19}$. Thus for the production of well defined equi-axed duplex structures in medium carbon low alloy steels, M300 and M500 initial microstructures are not recommended.

\section{Conclusion}

The influence of microstructural variables and processing conditions on the transformation behaviour of medium carbon low alloy steel during intercritical annealing treatment has been investigated. From the results, the following conclusions are drawn:

- The M300 samples had the slowest transformation rates in comparison to the M500 and the M650 initial microstructures.

- Transformation rates are generally faster for specimens with heavier prior deformation particularly at 740 and $760{ }^{\circ} \mathrm{C}$; at $780{ }^{\circ} \mathrm{C}$ the effect of degree of deformation was less obvious.

- The Transformation sequence was in four stages for the specimens treated at 740 and $760{ }^{\circ} \mathrm{C}$, while at $780{ }^{\circ} \mathrm{C}$ mainly two transformation patterns were observed - a five stage and a three stage transformation sequence.

- The M300 and M500 structures yielded inequigranular grain structures at peak hardness making them unsuitable for production of duplex structures with the potentials to optimize high strength and plasticity in comparison to the M650 structure.

\section{Acknowledgement}

The author is immensely grateful to the Association of Common Wealth Universities (ACU) for the award of its prestigious Wington Titular Fellowship in Engineering which was utilized at the Department of Materials Engineering, Indian Institute of Science, Bangalore, India. The mentoring received from Prof. S. Ranganathan and Prof. U. Ramamurty is immensely appreciated.

\section{References}

1. Ahmad E, Manzoor T and Hussain N. Thermomechanical processing in the intercritical region and tensile properties of dual-phase steel. Materials Science and Engineering A (Lausanne) 2009; 508(1-2):259-265.

2. Alaneme KK and Kamma CM. Evaluation of the influence of intercritical treatment variables on the transformation behaviour of a carbon steel. Abuja: Technical Transactions of The Nigerian Society of Engineers; 2009.

3. Alaneme KK. Evaluation of the phase transformation behaviour and mechanical properties of duplex microstructures in carbon steel. [thesis] Akure: Federal University of Technology.

4. Alaneme KK. Recrystallization studies of a low alloy steel in the $(a+\gamma)$ phase region. [dissertation]. Akure: Federal University of Technology; 2002.

5. American Society For Microbiology . Metallography and Microstructure. Washington, DC, 1998. (Handbook, 9).

6. Bag A, Ray KK and Dwarakadasa ES. Influence of martensite content and morphology on tensile and impact properties of high -martensite dualphase steels. Metallurgical and Materials Transactions A (Warrendale) 1991; 30(5):1193-1202.

7. Cota AB, Oliveira FLG, Barbosa AL, Lacerda CAM and Araujo FG. Microstructure and mechanical properties of a micro-alloyed steel after thermal treatments. Materials Research (Pittsburgh) 2003; 6(2):117-121. 
8. Erdogan M. Effect of austenite dispersion on phase transformation in dual phase steel. Scripta Materialia (Bethesda) 2003; 48(5):501-506.

9. Ghosh SK, Bandyopadhyay NR and Chattopadhyay PP. Effects of finish rolling temperature on the microstructures and mechanical properties of as hot rolled cu-added Ti, B micro-alloyed dual phase steels. Journal of The Institution of Engineers (I) (Sydney) 2004; 85:41-48.

10. Gorelik SS. Recrystallization in metals and alloys. $2^{\text {nd }}$ ed. Moscow: Mir Publishers; 1981.

11. Grajcar A. Effect of hot-working in the $\gamma+\alpha$ range on a retained austenite fraction in TRIP-aided steel. Journal of Achievements in Materials and Manufacturing Engineering (Gliwice) 2007; 22(2):79-82.

12. Guo Z, Sha W, Wilson EA and Grey RW. Improving toughness of PH13-8 stainless steel through Intercritical annealing. ISIJ International (Tokyo) 2003; 43(10):1622-1629.

13. Hansen N. New discoveries in deformed metals. Metallurgical and Materials Transactions A (Warrendale) 2001; 32A(12):2917-2919.

14. Holm EA, Miodownik MA and Rollet AD. On abnormal subgrain growth and the origin of recrystallization nuclei. Acta Materialia (Oxford) 2003; 51(9):2701-2716.

15. Kamma CM and Hornbogen E. Recrystallization mechanism in carbon steels. Canadian Metallurgical Quarterly (Otawa) 1984; 23(2):249-257.

16. Kamma CM. Optimum conditions for grain refinement in carbon steel by thermomechanical treatment. Materials Forum (Australia) 1990; 14:62-66.

17. Martin JW, Doherty RD and Cantor B. Stability of microstructure in metallic systems. $2^{\text {nd }}$ ed. Cambridge: Cambridge University Press; 1997.

18. Mazinani M and Poole WJ. Deformation behaviour of martensite in a low-carbon dual - phase steel. Advanced Materials Research (New York) 2007; 15-17:774-779.

19. Ovri $\mathrm{H}$ and Kamma CM. Evaluation of the transformation mechanisms and mechanical properties of ferrite - martensite microalloyed steels. Materials Research (Pittsburgh) 2008; 11(1):97-101.
20. Podder AS, Bhattacharjee D and Ray RK. Effect of martensite on the mechanical behaviour of ferrite-bainite dual phase steels. The Iron and Steel Institute of Japan (Tokyo) 2007; 47(7):1058-1064.

21. Rios PK, Siciliano Jr. F, Sandim HRZ, Plaut RL, and Padilha AF. Nucleation and growth during recrystallization. Materials Research (São Carlos) 2005; 8(3):225-238.

22. Rocha RO, Melo TMF, Pereloma EV and Santos DB. Microstructural Evolution at the initial stages of continuous annealing of cold rolled dual-phase steel. Materials Science and Engineering A (Lausanne) 2005; 391(1-2):296-304.

23. Salehi AR, Serajzadeh S, and Taheri AK. A Study on the microstructural changes in hot rolling of dual - phase steels. Journal of Materials Science (Norwell) 2006; 41(7):1917:1925.

24. Speer JG and Matlock DK. Constitutive behavior of high strength multiphase sheet steels under high strain rate deformation conditions. AISI-Technology Program, American Iron and Steel Institute; 2005.

25. Stuwe HP, Padilha AF and Siciliano Jr F. Competition between recovery and recrystallization. Materials Science and Engineering A (Lausanne) 2002; 233(1-2):361-367.

26. Sun $\mathrm{S}$ and Pugh M. Properties of thermomechanically processed dual-phase steels containing fibrous Martensite. Materials Science and Engineering A (Lausanne) 2002; 335(1):298-308.

27. Sun S, and Pugh M. Properties of thermomechanically processed dual - phase steels containing fibrous martensite. Materials Science and Engineering A (Lausanne) 2002; 335(1-2):298-308.

28. Torres RCE, Sanchez FH, Gonzalez A, Actis F and Herrera R. Study of the kinetics of the recrystallization of cold-rolled low carbon steel. Metallurgical Materials Transactions A (Warrendale) 2002; 32(1):25-31.

29. Xiaoyan S, Markus R, Clemens M and Hans E. Modelling of recrystallization after inhomogenous deformation. Metallurgical Materials Transactions A (Warrendale) 2001; 32(9):2199-2206.

30. Zeytin HK, Kubilay $\mathrm{C}$ and Aydin H. Investigation of dual phase transformation of commercial low alloy steels: effect of holding time at low inter-critical annealing temperatures. Materials Letters (Amsterdam) 2008; 62(17-18):2651-2653. 